\title{
On square permutations
}

\author{
Enrica Duchi and Dominique Poulalhon
}

Laboratoire d'Informatique Algorithmique: Fondements et Applications CNRS UMR 7089, Université Paris DiderotParis 7, Case 701475205 Paris Cedex 13

Severini and Mansour introduced square polygons, as graphical representations of square permutations, that is, permutations such that all entries are records (left or right, minimum or maximum), and they obtained a nice formula for their number. In this paper we give a recursive construction for this class of permutations, that allows to simplify the derivation of their formula and to enumerate the subclass of square permutations with a simple record polygon. We also show that the generating function of these permutations with respect to the number of records of each type is algebraic, answering a question of Wilf in a particular case.

\section{Introduction}

A permutation $\pi=\pi_{1} \ldots \pi_{n}$ of size $n$ can be naturally represented on $\mathbb{Z}^{2}$ by the set $\mathcal{G}_{\pi}$ of points at coordinates $\left(i, \pi_{i}\right)$. In order to construct a grid polygon on $\mathcal{G}_{\pi}$, we classify its elements as follows: a point $(i, j) \in \mathcal{G}_{\pi}$ is respectively said to be

- a left-right minimum (lrmin) if, for any $\left(i^{\prime}, j^{\prime}\right) \in \mathcal{G}_{\pi}, i^{\prime}<i \Longrightarrow j^{\prime}>j$;

- a right-left minimum (rlmin) if, for any $\left(i^{\prime}, j^{\prime}\right) \in \mathcal{G}_{\pi}, i^{\prime}>i \Longrightarrow j^{\prime}>j$;

- a left-right maximum (lrmax) if, for any $\left(i^{\prime}, j^{\prime}\right) \in \mathcal{G}_{\pi}, i^{\prime}<i \Longrightarrow j^{\prime}<j$;

- a right-left maximum (rlmax) if, for any $\left(i^{\prime}, j^{\prime}\right) \in \mathcal{G}_{\pi}, i^{\prime}>i \Longrightarrow j^{\prime}<j$;

- an interior point otherwise.

Observe that these definitions agree with usual definitions of lrmin, rlmin, lrmax, rlmax on permutations.

The sequences of lrmin, rlmin, lrmax and rlmax are respectively called left lower path, right lower path, left upper path and right upper path of $\mathcal{G}_{\pi}$. The record polygon of $\pi$ is the grid polygon with the concatenation of its left lower path, right lower path, right upper path and left upper path as contour.

A square permutation is a permutation without interior point, and a square polygon is the record polygon of such a permutation. Square polygons were defined in a slightly different but equivalent way by Severini and Mansour [6]. They are also related to the permutominoes considered by Boldi et al. [2] and Rinaldi et al. [4].

An extremal point is a point on the border of the bounding box of the polygon. A descending double point is a non extremal point that is simultaneously lrmin and rlmax. An ascending double point is a non 1365-8050 (c) 2008 Discrete Mathematics and Theoretical Computer Science (DMTCS), Nancy, France 
extremal point that is simultaneously lrmax and rlmin. These points are respectively the common points between the left lower and right upper paths, and between the left upper and right lower paths. Points of the polygon that are neither extremal nor double are said simple. A simple square polygon is a square polygon without double points. Observe that ascending double points of a record polygon are fixed points of the associated permutation, and descending double points are fixed points of the reverse permutation; moreover, a non simple square polygon has either descending or ascending double points, but not both, and these two classes of square polygons are mapped one onto the other by vertical symmetry.

From now on we denote indifferently by $\pi=\pi_{1} \pi_{2} \ldots \pi_{n}$ a square permutation or the associated square polygon, and by $\mathcal{S P}{ }_{n}$ the class of square permutations (or square polygons) of size $n$.

In Section 2 we present a construction for square permutations of size $n$. Our construction allows us in Section 2.3 to recover easily the formula obtained by Severini and Mansour, but also to control various parameters in Section 3: we show in particular that their generating function according to the parameters lrmin, lrmax, rlmin and rlmax is algebraic. This answers in the special case of square permutations a question of Wilf during an invited communication at CRM [7], asking for a way yo keep track simultaneously of these four types of records in permutations. Finally our construction allows us to calculate the number of square permutations with a simple record polygon.

\section{A construction for the class of square polygons}

\subsection{The construction}

We now define some operations to produce unambiguously all permutations in class $\mathcal{S P}_{n+1}$ out of permutations in class $\mathcal{S P} \mathcal{P}_{n}$.

In order to define these operations, we introduce the following notations. Given a square permutation $\pi$, let $j_{\pi}$ denote the least integer such that $\left(j_{\pi}, \pi_{j_{\pi}}\right)$ is a simple point belonging to the left lower path of $\pi$. Observe that this point is well defined except for permutations without simple points in their left lower path, in which case we define $j_{\pi}$ as the absissa of its extremal point with ordinate 1 . Then the points of left lower path with absissa strictly between 1 and $j_{\pi}$ form a (possibly empty) sequence of $\ell(\pi)$ descending

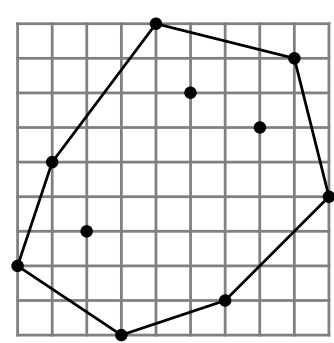

(a) An ordinary one,

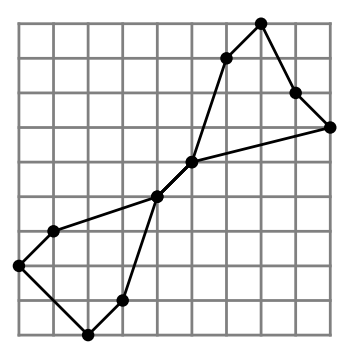

(b) a square one, with ascending double points

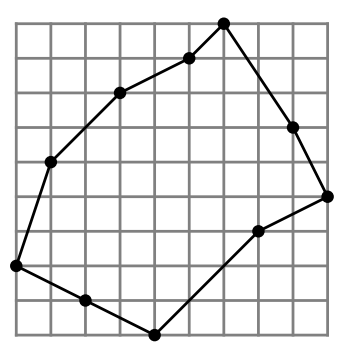

(c) and a simple square one.

Fig. 1: Some examples of record polygons. 
double points which we call the initial double points of $\pi$. Let also define the following interval:

$H(\pi)=\left\{\begin{array}{ll}\llbracket \pi_{j_{\pi}}+1, \pi_{1} \rrbracket & \text { if } \pi_{j_{\pi}} \neq 1, \\ \llbracket 1, \pi_{1} \rrbracket & \text { if } \pi_{j_{\pi}}=1,\end{array} \quad\right.$ with cardinality $\quad h(\pi)=|H(\pi)|= \begin{cases}\pi_{1}-\pi_{j_{\pi}} & \text { if } \pi_{j_{\pi}} \neq 1, \\ \pi_{1} & \text { if } \pi_{j_{\pi}}=1 .\end{cases}$

We are now in position to define the construction. For any $p \in H(\pi)$, let $\vartheta_{1}^{p}(\pi)$ and $\vartheta_{2}^{p}(\pi)$ be respectively the permutations $\sigma=\sigma_{1} \ldots \sigma_{n+1}$ and $\tau=\tau_{1} \ldots \tau_{n+1}$ defined by:

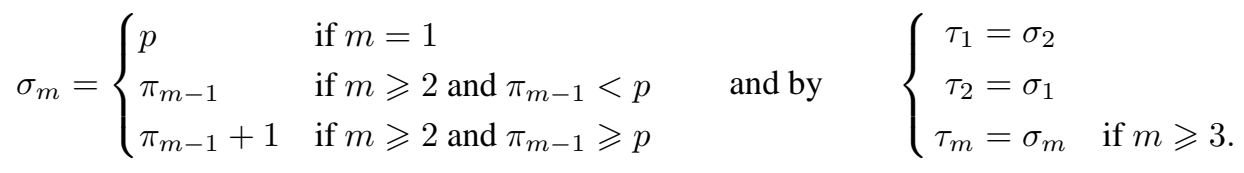

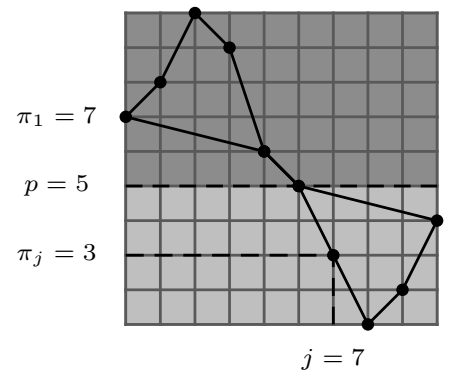

(a) A permutation $\pi$,

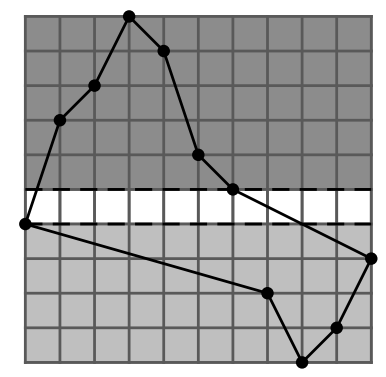

(b) $\vartheta_{1}^{p}(\pi)$,

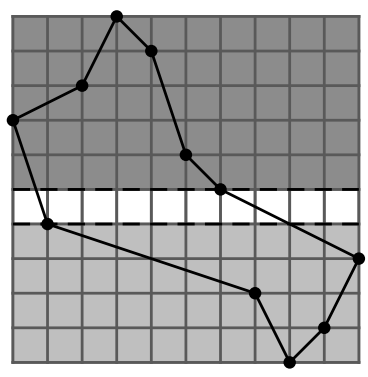

(c) and $\vartheta_{2}^{p}(\pi)$.

Fig. 2: Example of the $\vartheta$-construction

This construction is illustrated in Figure 2. In other terms, given a polygon $\pi$ and an integer $p$ in the interval $H(\pi)$, we produce a polygon $\vartheta_{1}^{p}(\pi)$ by inflating the polygon between lines $p-1$ and $p$ to insert a point in a new first column, and a polygon $\vartheta_{2}^{p}(\pi)$ by exchanging the first two columns of the previous polygon. Finally let $\vartheta(\pi)$ be the set of all $\vartheta_{i}^{p}(\pi)$ for $i$ in $\{1,2\}$ and $p$ in $H(p)$. This set has clearly cardinality $2 h(\pi)$.

Theorem 1 All elements of $\mathcal{S} \mathcal{P}_{n+1}$ are produced in a unique manner by applying the operator $\vartheta$ to all objects in $\mathcal{S P}_{n}$ :

$$
\mathcal{S P}_{n+1}=\bigsqcup_{\pi \in \mathcal{S} \mathcal{P}_{n}} \vartheta(\pi)
$$

Proof: Omitted.

Observe that $\vartheta$ forms an ECO operator in the sense of [1], that is, it gives a way of producing each square permutation of size $n+1$ from exactly one square permutation of size $n$, and this recursive construction translates into equations for the generating function of square permutations, which we exploit in the newt sections. Figure 3 shows the first levels of the generating tree associated with the previous construction. 


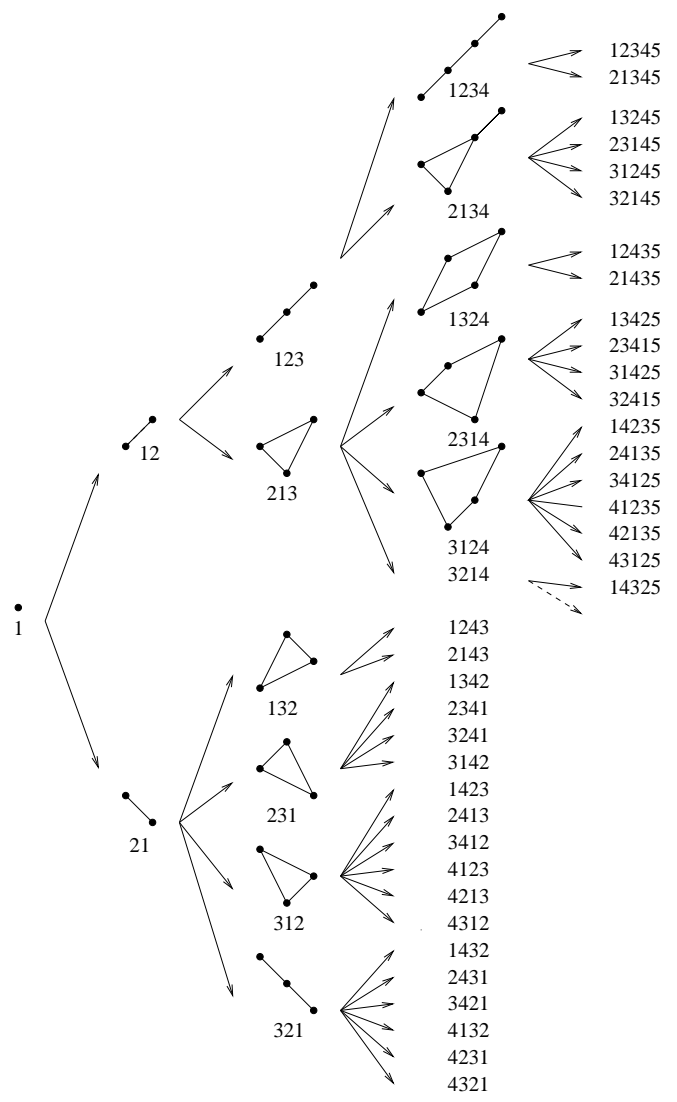

Fig. 3: The first levels of the generating tree of square permutations.

\subsection{The succession rule}

Now we want to describe the way the construction $\vartheta$ affects the parameter $h$. This leads us to distinguish four different sub-classes $\mathcal{A}_{n}, \mathcal{B}_{n}, \mathcal{C}_{n}$ and $\mathcal{D}_{n}$ of $\mathcal{S} \mathcal{P}_{n}$, depending on the values of $\pi_{1}$ and $\pi_{j_{\pi}}$ :

\begin{tabular}{c|c|c|} 
& $\pi_{1}=n$ & $\pi_{1}<n$ \\
\hline$\pi_{j_{\pi}}=1$ & $\mathcal{A}$ (Fig. 4(a)) & $\mathcal{B}$ (Fig. 4(b)) \\
\hline$\pi_{j_{\pi}}>1$ & $\mathcal{C}$ (Fig. 4(c)) & $\mathcal{D}$ (Fig. 4(d)) \\
\hline
\end{tabular}

Figure 4 shows the shapes of generic square permutations in each subclass, with a non-empty initial sequence of double points, but of course this sequence may be empty.

For each one of the previous classes, we want to describe the evolution of the parameter $h$ (the label in ECO-terminology) by giving a so-called succession rule, which describes the types and labels of the polygons generated out of a polygon with a given type and label. 


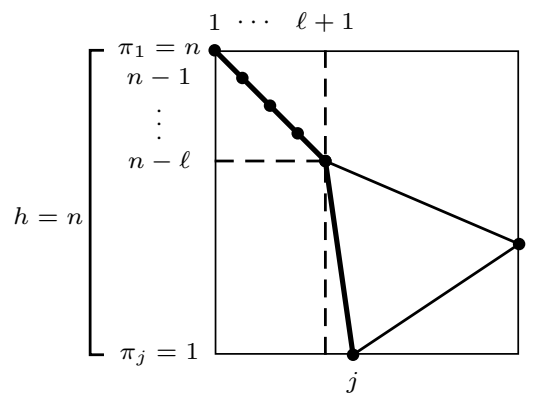

(a) Class $\mathcal{A}$,

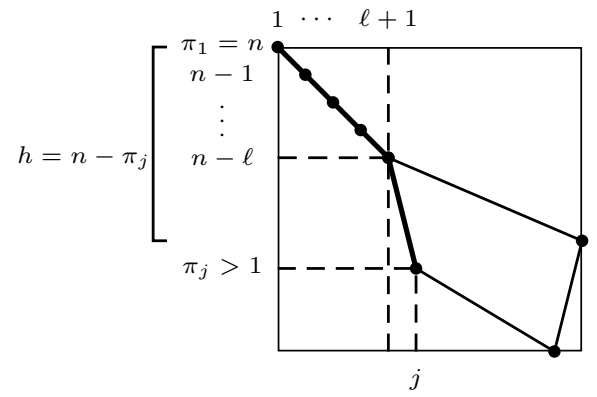

(c) class $\mathcal{C}$,

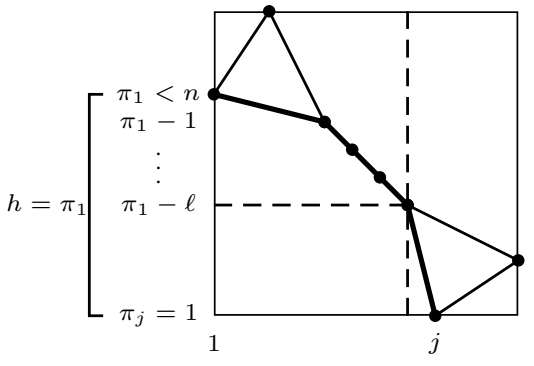

(b) class $\mathcal{B}$,

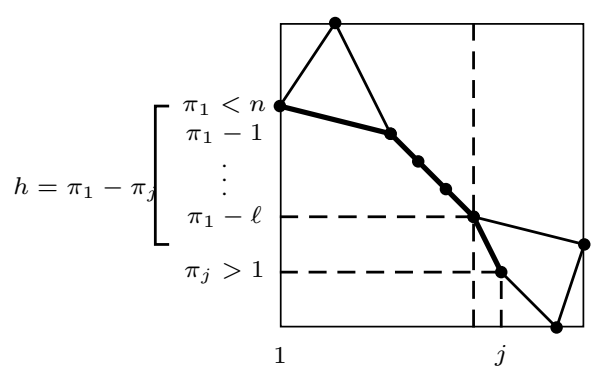

(d) and class $\mathcal{D}$.

Fig. 4: The four subclasses of square polygons (cases of a non-empty initial sequence of double points).

\subsubsection{Class $\mathcal{A}$, illustrated in Figure 5}

For any polygon $\pi \in \mathcal{A}_{n}, h(\pi)=n$ and the construction consists in adding a point with ordinate $p$, $1 \leqslant p \leqslant n$, in the first or second column of the polygon. In the picture we distinguish the cases in which the added point has ordinate $p=1,1<p<n$ or $p=n$.

The first operation $\vartheta_{1}$ produces only polygons $\sigma$ of type $\mathcal{B}$ since $\sigma_{1}=p<n+1$, and 1 remains the ordinate of the first simple point in the left lower path of $\sigma$ (in other words $\sigma_{j_{\sigma}}=1$ ); for these polygons, $h(\sigma)=p$.

The second operation $\vartheta_{2}$ produces polygons $\tau$ of type $\mathcal{A}$ for $p=1$ and $p=n$, since in these cases $\tau_{1}=n+1$, and 1 remains the ordinate of the first simple point in the left lower path of $\tau$. Hence for both of these cases $h(\tau)=n+1$. For $1<p<n$, the second operation produces a polygon $\tau$ of type $\mathcal{C}$ since $\tau_{2}=p$ is the first simple point in the left lower path of $\tau$. For these cases $h(\tau)=n+1-p$.

Then this can be summarized by a succession rule as follows:

$$
(n)_{\mathcal{A}} \rightarrow(n+1)_{\mathcal{A}}^{2}(1)_{\mathcal{B}}(2)_{\mathcal{B}} \ldots(n)_{\mathcal{B}}(2)_{\mathcal{C}}(3)_{\mathcal{C}} \ldots(n-1)_{\mathcal{C}}
$$

meaning that a polygon of type $\mathcal{A}$ and label $n$ produces:

- 2 polygons of type $\mathcal{A}$ with labels $n+1$, 


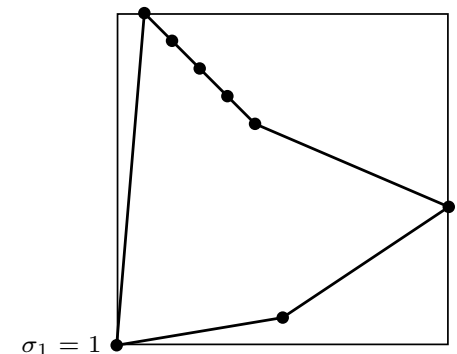

(a) $\vartheta_{1}^{1}(\pi) \in \mathcal{B}$, with label 1 ,

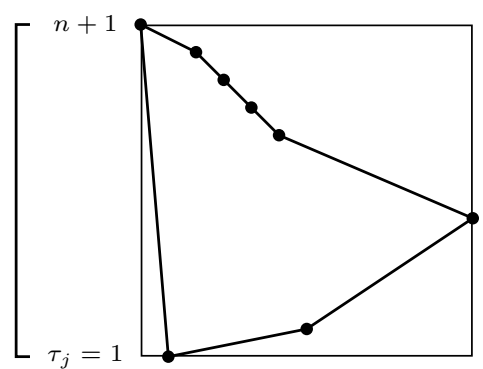

(d) $\vartheta_{2}^{1}(\pi) \in \mathcal{A}$, with label $n+1$,

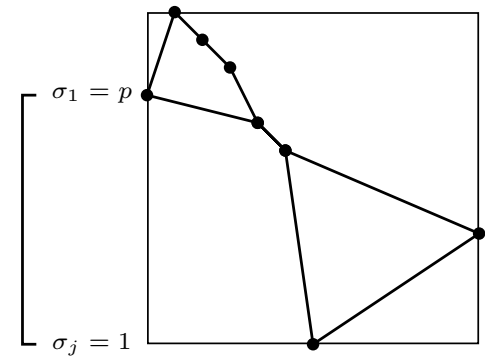

(b) $\vartheta_{1}^{p}(\pi) \in \mathcal{B}$, with label $p$,

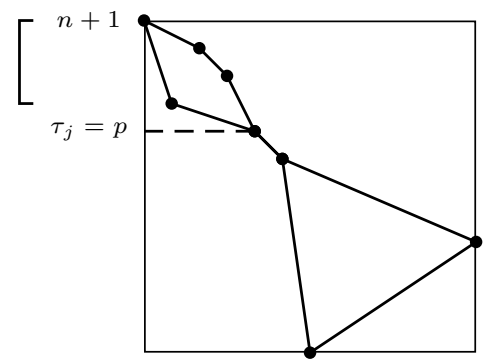

(e) $\vartheta_{2}^{p}(\pi) \in \mathcal{C}$, with label $n+1-p$,

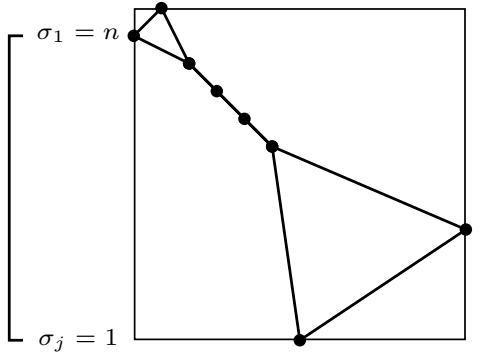

(c) $\vartheta_{1}^{n}(\pi) \in \mathcal{B}$, with label $n$,

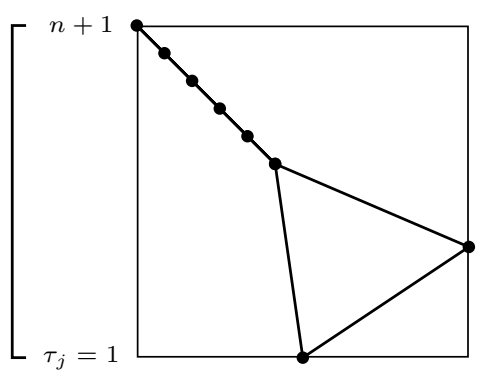

(f) $\vartheta_{2}^{n}(\pi) \in \mathcal{A}$, with label $n+1$.

Fig. 5: Construction for polygons of class $\mathcal{A}$ (with shape as in Fig. 4(a)), in the three cases $p=1,1<p<n$ and $p=n$.

- $n$ polygons of type $\mathcal{B}$ with respective labels $1,2, \ldots, n$,

- $n-2$ polygons of type $\mathcal{C}$ with respective labels $2,3, \ldots, n-1$.

\subsubsection{Class $\mathcal{B}$, illustrated in Figure 6}

Let $\pi \in \mathcal{B}_{n}$; observe that $h(\pi)=\pi_{1}$. In Figure 6 we distinguish the case $p=1$ and the generic case $1<p \leqslant k$.

The first operation $\vartheta_{1}$ produces polygons $\sigma$ of type $\mathcal{B}$ since $\sigma_{1} \neq n+1$, and 1 remains the ordinate of the first simple point in the left lower path. For these polygons $h(\sigma)=p$.

The second operation produces for $p=1$ a polygon $\tau$ of type $\mathcal{B}$ since $\tau_{1} \neq n+1$, and 1 remains the ordinate of the first simple point in the left lower path. For this polygon $h(\tau)=h(\pi)+1$. The other polygons have type $\mathcal{D}$ since $\tau_{1} \neq n+1$ and $\tau_{2}=p>1$ is the ordinate of the first simple point in the left lower path. Hence $h(\tau)=h(\pi)+1-p$ for these polygons.

Therefore we have the following succession rule for this case:

$$
(h)_{\mathcal{B}} \rightarrow(1)_{\mathcal{B}}(2)_{\mathcal{B}} \ldots(h+1)_{\mathcal{B}}(1)_{\mathcal{D}}(2)_{\mathcal{D}} \ldots(h-1)_{\mathcal{D}}
$$




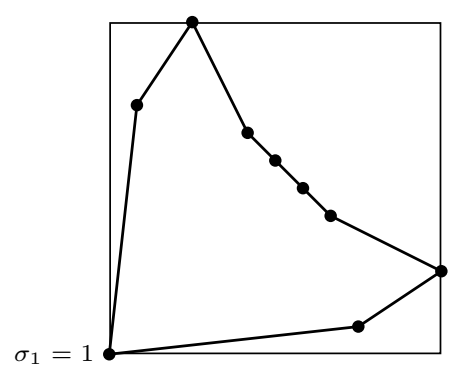

(a) $\vartheta_{1}^{1}(\pi) \in \mathcal{B}$, with label 1 ,

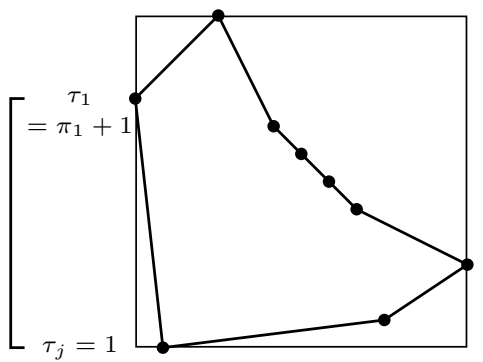

(c) $\vartheta_{2}^{1}(\pi) \in \mathcal{B}$, with label $h(\pi)+1$,

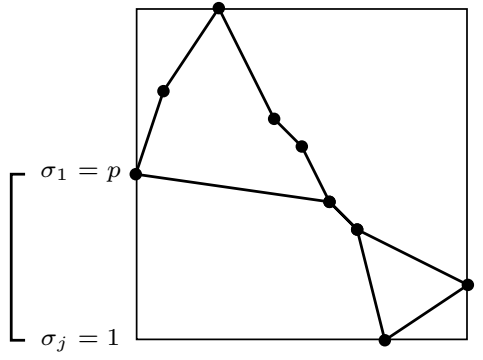

(b) $\vartheta_{1}^{p}(\pi) \in \mathcal{B}$, with label $p$,

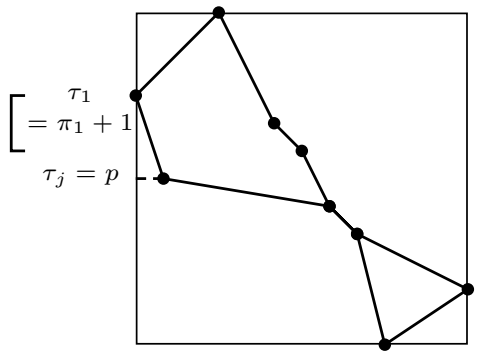

(d) $\vartheta_{2}^{p}(\pi) \in \mathcal{B}$, with label $h(\pi)+1-p$.

Fig. 6: The construction for polygons of type $\mathcal{B}$ (with shape as in Fig. 4(b)), in the two cases $p=1$ and $1<p \leqslant h(\pi)$.

\subsubsection{Class $\mathcal{C}$, illustrated in Figure 7}

Let $\pi \in \mathcal{C}_{n}$; observe that $1<h(\pi)<n-1$. We distinguish the case $p=n$ and the cases $n-h(\pi)<$ $p<n$.

The first operation $\vartheta_{1}$ produces in any case a polygon $\sigma$ of type $\mathcal{D}$, since $\sigma_{1} \neq n+1$ and $\pi_{j} \neq 1$ is the ordinate of the first simple point in the left lower path. Hence for these polygons $h(\sigma)=p-n+h(\pi)$.

The second operation $\vartheta_{2}$ produces only polygons of type $\mathcal{C}$. Indeed when $\tau_{2}=p=n$ then $\pi_{j} \neq 1$ remains the ordinate of the first simple point in the left lower path, and $h(\tau)=h(\pi)+1$ for this polygon. On the contrary, when $\tau_{2}=p<n$ then $p$ is the ordinate of the first simple point of the left lower path and $h(\tau)=n+1-p$ for these polygons. Therefore we have the following succession rule for this case:

$$
(h)_{\mathcal{C}} \rightarrow(2)_{\mathcal{C}}(3)_{\mathcal{C}} \ldots(h+1)_{\mathcal{C}}(1)_{\mathcal{D}}(2)_{\mathcal{D}} \ldots(h)_{\mathcal{D}}
$$

\subsubsection{Class $\mathcal{D}$, illustrated in Figure 8}

Let $\pi \in \mathcal{D}_{n}$. In this case, $\pi_{1}<n$ and $\pi_{j}>1$, hence $p$ may not be equal to 1 nor $n$.

The first operation $\vartheta_{1}$ produces polygons $\sigma$ of type $\mathcal{D}$ since $\sigma_{1}=p<n$ and the ordinate of the first simple point of the lower path is the same as for $\pi$. Hence $h(\sigma)=p-\pi_{1}+h(\pi)$. 


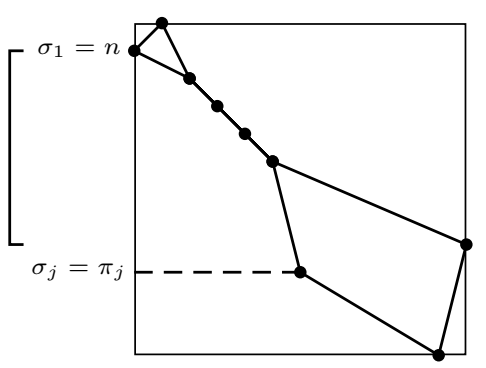

(a) $\vartheta_{1}^{n}(\pi) \in \mathcal{D}$, with label $h(\pi)$,

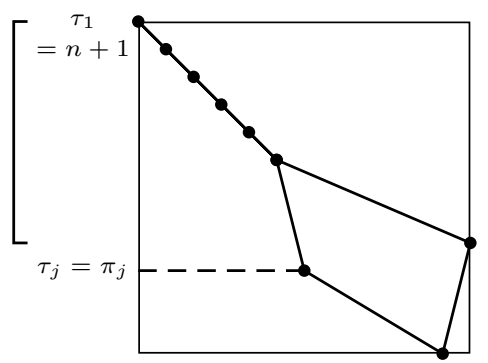

(c) $\vartheta_{2}^{n}(\pi) \in \mathcal{C}$, with label $h(\pi)+1$,

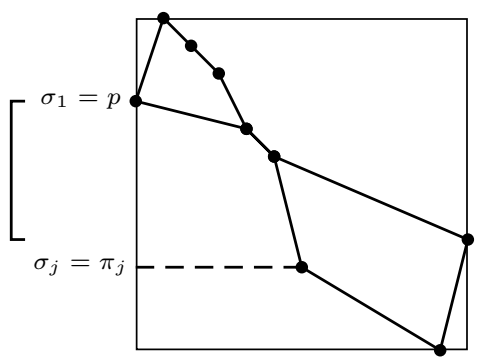

(b) $\vartheta_{1}^{p}(\pi) \in \mathcal{D}$, with label $p-n+h(\pi)$,

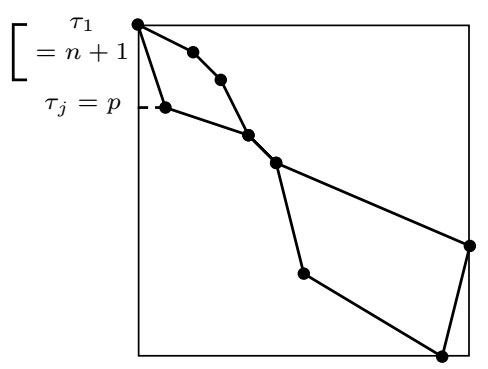

(d) $\vartheta_{2}^{p}(\pi) \in \mathcal{C}$, with label $n-p$.

Fig. 7: The construction for polygons of type $\mathcal{C}$ (with shape as in Fig. 4(c)), in the two cases $p=n$ and $n-h(\pi)<$ $p<n$.

The second operation $\vartheta_{2}$ also produces polygons $\tau$ of type $\mathcal{D}$ since $\tau_{1}=\pi_{1}+1 \neq n+1$ and $p \neq 1$ is the ordinate of the first simple point in the left lower path. Hence $h(\tau)=\pi_{1}+1-p$ for these polygons.

Therefore we have the following succession rule for this case:

$$
(h)_{\mathcal{D}} \rightarrow(1)_{\mathcal{D}}^{2}(2)_{\mathcal{D}}^{2} \ldots(h)_{\mathcal{D}}^{2}
$$

Finally, we remark that the permutation $\pi=1$ belongs to the class $\mathcal{A}$ and that the image of this permutation by the application $\vartheta$ consists of the permutation 12 , belonging to the class $\mathcal{B}$, and the permutation 21 , still belonging to the class $\mathcal{A}$. Then the complete succession rule is the following: 


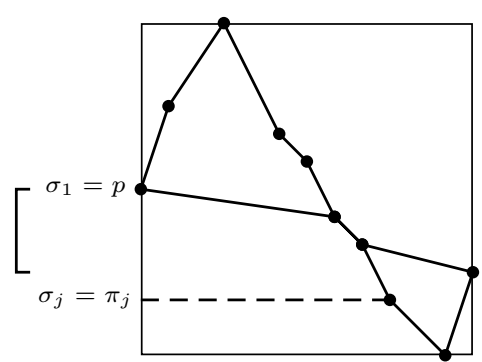

(a) $\vartheta_{1}^{p}(\pi) \in \mathcal{D}$, with label $p-\pi_{1}+h(\pi)$,

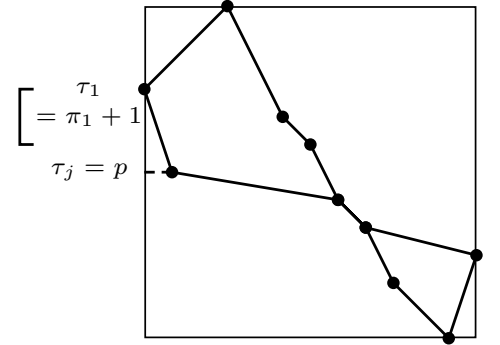

(b) $\vartheta_{2}^{p}(\pi) \in \mathcal{C}$, with label $\pi_{1}+1-p$.

Fig. 8: The construction for polygons of type $\mathcal{D}$ (with shape as in Fig. 4(d)), and $\pi_{1}-h(\pi)<p \leqslant \pi_{1}$.

$$
\left\{\begin{array}{lll}
(1)_{\mathcal{A}} & & \\
(1)_{\mathcal{A}} \rightarrow(2)_{\mathcal{A}}(1)_{\mathcal{B}} & \\
(h)_{\mathcal{A}} \rightarrow(h+1)_{\mathcal{A}}^{2}(1)_{\mathcal{B}}(2)_{\mathcal{B}} \ldots(h)_{\mathcal{B}}(2)_{\mathcal{C}}(3)_{\mathcal{C}} \ldots(h-1)_{\mathcal{C}} & \text { for } h \geqslant 2, \\
(h)_{\mathcal{B}} \rightarrow(1)_{\mathcal{B}}(2)_{\mathcal{B}} \ldots(h+1)_{\mathcal{B}}(1)_{\mathcal{D}}(2)_{\mathcal{D}} \ldots(h-1)_{\mathcal{D}} & \text { for } h \geqslant 1, \\
(h)_{\mathcal{C}} \rightarrow(2)_{\mathcal{C}}(3)_{\mathcal{C}} \ldots(h+1)_{\mathcal{C}}(1)_{\mathcal{D}}(2)_{\mathcal{D}} \ldots(h)_{\mathcal{D}} & \text { for } h \geqslant 1, \\
(h)_{\mathcal{D}} \rightarrow(1)_{\mathcal{D}}^{2}(2)_{\mathcal{D}}^{2} \ldots(h)_{\mathcal{D}}^{2} & \text { for } h \geqslant 1
\end{array}\right.
$$

\subsection{The generating function}

Proposition 1 Let $\mathcal{F}$ and $\mathcal{G}$ be classes of objects with size function $n$ and label $k$. Then any succession rule of the form $(k)_{\mathcal{F}} \rightarrow(1)_{\mathcal{G}} \ldots(k)_{\mathcal{G}}$ gives in the equation for the generating function $G(x, y)=$ $\sum_{o \in \mathcal{G}} x^{n(o)} y^{k(o)}$ of the class $\mathcal{G}$ a contribution

$$
\frac{x y}{1-y}[F(x, 1)-F(x, y)]
$$

where $F(x, y)=\sum_{o \in \mathcal{F}} x^{n(o)} y^{k(o)}$ is the generating function for the class $\mathcal{F}$.

More generally, a rule $(k)_{\mathcal{F}} \rightarrow(1+p)_{\mathcal{G}} \ldots(k+q)_{\mathcal{G}}$ for fixed integers $p$ and $q$ gives a contribution

$$
\frac{x y}{1-y}\left[y^{p} F(x, 1)-y^{q} F(x, y)\right] \text {. }
$$

Proof: The contribution to the generating function $G$ of objects generated at level $n+1$ from the objects at level $n$ by the rule $(k)_{\mathcal{F}} \rightarrow(1)_{\mathcal{G}} \ldots(k)_{\mathcal{G}}$ is:

$$
\sum_{o \in \mathcal{F}} \sum_{i=1}^{k(o)} x^{n(o)+1} y^{i},
$$


since each object $o$ with size $n(o)$ and label $k(o)$ produces $k(o)$ objects with size $n(o)+1$ and respective label $1, \ldots, k(o)$. Then this contribution rewrites as

$$
\sum_{o \in \mathcal{F}} x^{n(o)+1} \frac{y-y^{k(o)+1}}{1-y}=\frac{x y}{1-y}(F(x, 1)-F(x, y)) .
$$

According to this proposition we can easily translate the previous succession rule in the following system of functional equations for the generating functions of the classes $\mathcal{A}, \mathcal{B}, \mathcal{C}$ and $\mathcal{D}$. Let

$$
A(y)=\sum_{\pi \in \mathcal{A}} x^{n(\pi)} y^{h(\pi)},
$$

and define similarly $B(y), C(y)$, and $D(y)$ (we write explicitly only the variables that need to be substituted); then:

$$
\left\{\begin{array}{l}
A(y)=x^{2} y^{2}+2 x y A(y), \\
B(y)=x^{2} y^{1}+\frac{x y}{1-y}[A(1)-A(y)]+\frac{x y}{1-y}[B(1)-y B(y)], \\
C(y)=\frac{x y}{1-y}\left[y A(1)-y^{-1} A(y)\right]+\frac{x y^{2}}{1-y}[C(1)-C(y)], \\
D(y)=\frac{x y}{1-y}\left[B(1)-y^{-1} B(y)\right]+\frac{x y}{1-y}[C(1)-C(y)]+2 \frac{x y}{1-y}[D(1)-D(y)] .
\end{array}\right.
$$

From the previous system, we see that $A(y)$ is rational. Replacing $A(1)$ and $A(y)$ by their explicit expressions in the equation for $B(y)$ leads to an equation for the unknowns $B(y)$ and $B(1)$ that can be solved by the kernel method, that is, a method for solving linear equations with one catalitic variable [5, Chapter VII.8.1]. Doing the same thing for $C(y)$ and $D(y)$ we obtain the following expressions:

$$
\left\{\begin{array}{l}
A(1)=\frac{x^{2}}{1-2 x} \\
B(1)=\frac{x(x-1)}{1-2 x}+\frac{x}{\sqrt{1-4 x}} \\
C(1)=\frac{x^{2}}{1-2 x}+\frac{x}{\sqrt{1-4 x}}, \\
D(1)=x \frac{1-7 x+14 x^{2}-4 x^{3}}{(1-2 x)(1-4 x)^{2}}+\frac{x(1-3 x)}{(1-4 x)^{3 / 2}} .
\end{array}\right.
$$

Now the generating function for square polygons according to the number of points is given by

$$
A(1)+B(1)+C(1)+D(1)
$$

and this yields the result obtained by Severini and Mansour in [6].

Theorem 2 (Severini-Mansour) The generating function of square polygons according to the size is

$$
\frac{2 x^{2}(1-3 x)}{(1-4 x)^{2}}-\frac{4 x^{3}}{(1-4 x)^{\frac{3}{2}}} .
$$




\section{Refined enumeration}

\subsection{New parameters}

Square polygons consist in four paths, or faces, and we want to study the number of points in each of these paths: in other terms we consider the numbers of lrmin, rlmin, lrmax and rlmax in square permutations. Equivalently, we consider the number of edges in each faces rather than the number of points.

As we look for a formula for the number of simple square permutations, we also want to keep track of double points. Recall that polygons may have either descending or ascending double points, but not both, and that these two classes are mapped one onto the other by vertical symmetry. The parameters we are interested in are therefore the following: let $\pi$ be a square polygon and

- $\alpha(\pi)$ be the number of edges in the left lower path of $\pi$, minus the number of descending double points.

- $\beta(\pi)$ be the number of edges in the left upper path of $\pi$,

- $\gamma(\pi)$ be the number of edges in the right upper path of $\pi$, minus the number of descending double points.

- $\delta(\pi)$ be the number of edges in the right lower path of $\pi$,

As before, let $\ell(\pi)$ denote the number of initial double points, $h(\pi)=k(\pi)+\ell(\pi)$, and $m(\pi)$ be the number of non initial double points: we need to refine the parameter $h$ of our first succession rule by distinguishing the contribution of double points in the active region.

\subsection{The refined succession rule}

The recursive construction given in Section 2 allows to follow these new parameters. Consider for instance the case of a square polygon $\pi$ of type $\mathcal{A}$; as seen before, $\tau=\vartheta_{2}^{1}(\pi)$ is a square polygon of type $\mathcal{A}$; but this can be specified: $\tau$ has no initial double points, its left lower path is reduced to the segment $[(1, n+1),(2,1)]$, its other double points are (the translated of) $\pi$ 's ones, and initial double points of $\pi$ become single points of $\tau$ 's right upper path. This gives the following contribution to the succession rule, according to the refined multilabel $(k, \ell, m, \alpha, \beta, \gamma, \delta)$ :

$$
(k, \ell, m, \alpha, 0, \gamma, \delta)_{\mathcal{A}} \rightarrow(k+\ell+1,0, m, 1,0, \gamma+\ell, \delta+1)_{\mathcal{A}}
$$


Reconsidering all the special cases leads to the following complete succession rule (in sequences of multilabels of the same shape, bold symbols are used to emphasize parameters that fluctuate):

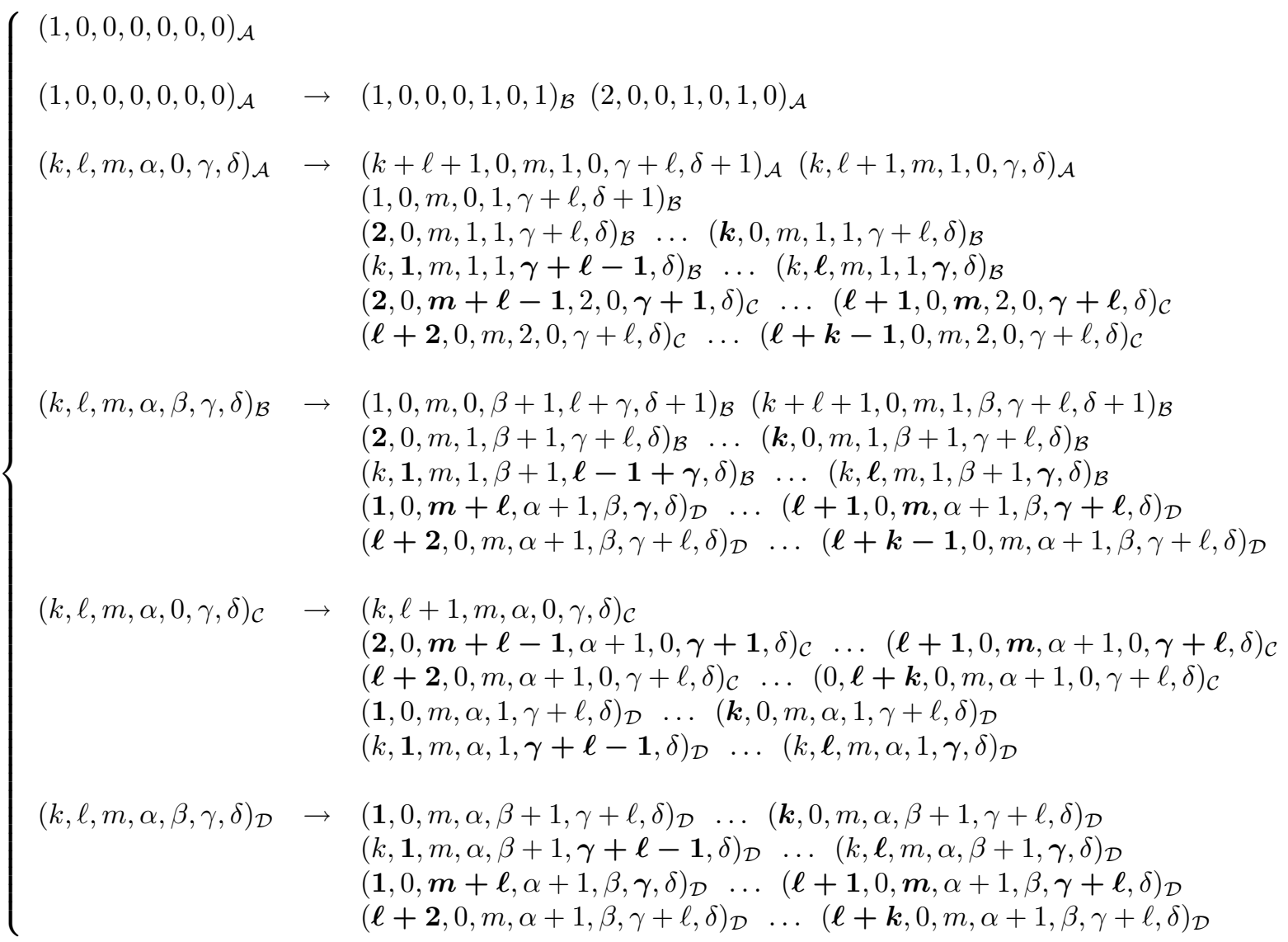

\subsection{The generating function}

Proposition 2 Any succession rule of type $(p, q)_{\mathcal{F}} \rightarrow(1, p+q-1)_{\mathcal{G}} \ldots(p, q)_{\mathcal{G}}$ gives in the equation for the associated generating function $G(y, z)=\sum_{o \in \mathcal{G}} x^{n(o)} y^{p(o))} z^{q(o)}$ of the class $\mathcal{G}$ a contribution

$$
x \frac{y}{z-y}[F(z, z)-F(y, z)]
$$

where $F(y, z)=\sum_{o \in \mathcal{F}} x^{n(o)} y^{p(o)} z^{p(o)}$ is the generating function for the class $\mathcal{F}$. 
Proof: According to the rule $(p, q) \rightarrow(1, p+q-1) \ldots(p, q)$ we have that:

$$
\begin{aligned}
\sum_{o \in \mathcal{F}} \sum_{i=1}^{p(o)} x^{n(o)+1} y^{i} z^{p(o)+q(o)-i} & =\sum_{o \in \mathcal{F}} x^{n(o)+1} z^{p(o)+q(o)} \sum_{i=1}^{p(o)}\left(\frac{y}{z}\right)^{i} \\
& =\sum_{o \in \mathcal{F}} x^{n(o)+1} z^{p(o)+q(o)} \frac{\frac{y}{z}-\left(\frac{y}{z}\right)^{p(o)+1}}{1-\frac{y}{z}}
\end{aligned}
$$

That is: the contribution to the class $\mathcal{G}$ is given by $x \frac{y}{z-y} F(z, z)-\frac{y}{z-y} F(y, z)$.

Proposition 3 Any succession rule of type $(p, q, r, s)_{\mathcal{F}} \rightarrow(0,1, p+r, s)_{\mathcal{G}} \ldots(0, p+1, r, s+p)_{\mathcal{G}}$ gives in the equation for the associated generating function $G(y, z)=\sum_{o \in \mathcal{G}} x^{n(o)} y^{p(o)} z^{q(o)} w^{r(o)} t^{s(o)}$ of the class $\mathcal{G}$ a contribution

$$
x \frac{z}{w-t z}[w F(w, 1, w, t)-t z F(t z, 1, w, t)],
$$

where $F(y, z, w, t)=\sum_{o \in \mathcal{F}} x^{n(o)} y^{p(o)} z^{q(o)} w^{r(o)} t^{s(o)}$ is the generating function for the class $\mathcal{F}$.

Proof: Omitted.

Let

$$
A(y, z, u, v)=\sum_{o \in \mathcal{A}} x^{n(o)} y^{l(o)} z^{k(o)} w^{m(o)} u^{\alpha(o)} v^{\beta(o)} s^{\gamma(o)} t^{\delta(o)}
$$

where we only indicate variables we substitute in the right-hand side of the equations, and similarly for $\mathcal{B}, \mathcal{C}$ and $\mathcal{D}$. According to the previous propositions we can easily translate the refined succession rule in the following system of functional equations: 


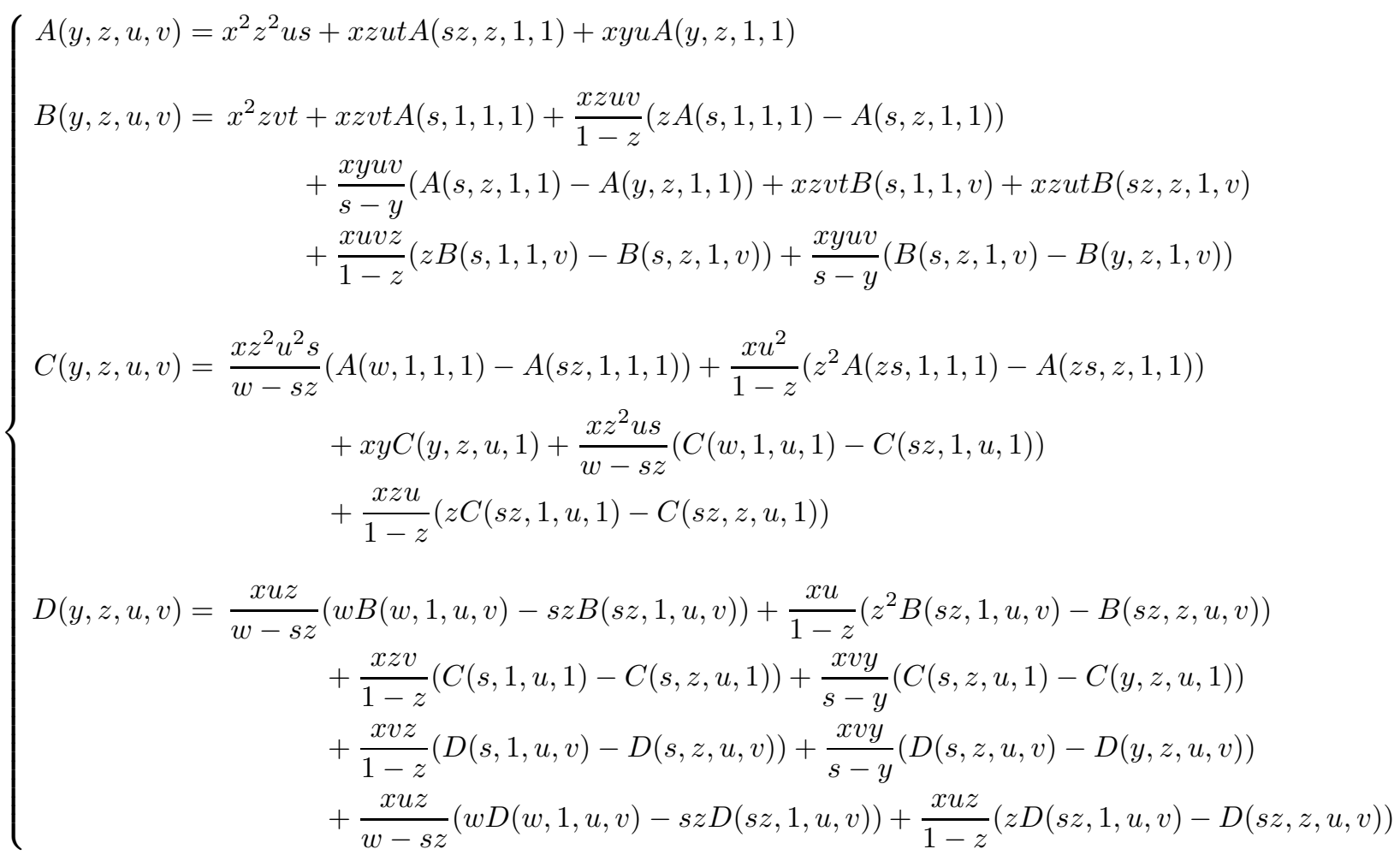

Theorem 3 The series $\mathcal{A}, \mathcal{B}, \mathcal{C}$ and $\mathcal{D}$ are algebraic series.

\section{Proof:}

- Setting $y=s z$ in the first equation we obtain $A(s z, z, 1,1)$ and then $A(y, z, 1,1)$ and $A(y, z, u, v)$, all are rational in the variables.

- Setting $y=s z$ and $u=1$, the terms in $B(s, z, 1, v)$ cancel and it remains an equation between $B(s z, z, 1, v)$ and $B(s, 1,1, v)$ which is solved by the kernel method for variable $z$. Then we apply the kernel method for variable $y$ to the original equation with $u=1$, to get $B(y, z, 1, v)$ and $B(s, z, 1, v)$. Finally returning again to the original equation we get $B(y, z, u, v)$ which is rational in the variables and the Catalan refinement:

$$
Z_{0}(x ; v, t)=\frac{1-x v+x t-\sqrt{(1-x v+x t)^{2}-4 x t}}{2 x t} .
$$

- We subtract to the third equation with $v=1$, the same equation with $v=1$ and $y=s z$, to get an equation between $C(y, z, u, 1)$ and $C(s z, z, u, 1)$. Setting $y=w$ and $z=1$ in this equation gives $C(w, 1, u, 1)$ in terms of $C(s, 1, u, 1)$. Setting instead $z=1$ and $y=s z$ in this equation 
gives $C(s z, 1, u, 1)$ in terms of $C(s, 1, u, 1)$. With these equation the original equation is rewritten in terms of $C(s z, z, u, 1)$ and $C(s, 1, u, 1)$ and the kernel method for variable $z$ allows to express these series, and then $C(y, z, u, v)$ as rational functions of the variables and

$$
Z_{0}(x ; u, s)=\frac{1-x u+x s-\sqrt{(1-x u+x s)^{2}-4 x s}}{2 x s} .
$$

- We subtract to the fourth equation, the same equation with $y=s z$, to get an equation (*) between $D(y, z, u, v), D(s z, z, u, v)$ and $D(s, z, u, v)$. Applying the kernel method for the variable $y$ then gives $D(s, z, u, v)$ in terms of $D(s z, z, u, v)$, which allows to rewrite (*) as an equation between $D(y, z, u, v)$ and $D(s z, z, u, v)$ in which we can set $z=1$ (the terms of the form $\frac{1}{1-z}$ have cancelled). We then obtain $D(s, 1, u, v), D(y, 1, u, v)$ and $D(y, z, u, v)$ as rational functions of the variables, $Z_{0}(x ; v, t)$ and $Z_{0}(x ; u, s)$.

\section{Counting simple square polygons}

Proposition 4 The number $s_{n}$ of simple square polygons of size $n$ is

$$
s_{n}=p_{n}-2\left(p_{n}-q_{n}\right),
$$

where $p_{n}$ is the number of square polygons of size $n$, and $q_{n}$ is the number of square polygons without descending double points.

Proof: $p_{n}-q_{n}$ is the number of square polygons with at least one descending double point, and also by symmetry, the number of square polygons with at least one ascending double point. Since a square polygon cannot have both ascending and descending double points the result follows.

Let $\mathcal{P}=\mathcal{A}+\mathcal{B}+\mathcal{C}+\mathcal{D}$ be the generating function of all polygons. Then the generating function of $q_{n}, \sum_{n} q_{n} x^{n}$ with respect to the size is obtained by putting $y=0$ and $w=0$ in $\mathcal{P}$ to select polygons without double points, and the other variables to 1 to forget the other parameters. This gives:

$$
\frac{1}{2(2+x)}\left(\frac{x^{2}(8-23 x)}{(1-4 x)^{2}}-\frac{x^{3}(17+4 x)}{(1-4 x)^{3 / 2}}\right) .
$$

Theorem 4 The generating function of simple square polygons with respect to the size is

$$
\frac{1}{2+x}\left(\frac{x^{2}\left(4-13 x+6 x^{2}\right)}{(1-4 x)^{2}}-\frac{9 x^{3}}{(1-4 x)^{3 / 2}}\right) .
$$

\section{Conclusion}

In this paper we presented a recursive construction for the class of square permutations. This led us to count these permutations with respect to their size, recovering a result of Severini and Mansour. Moreover we obtained the algebraic generating function of square permutations with respect to the number of 
records of each type (lrmin, lrmax, rlmin, rlmax). It would be interesting to extend the recursive construction to the class of all permutations, that is to construct square permutations with interior points and count them with respect to the four types of records. Moreover, since the number of square polygons according to their size is almost the same as the number of convex polyominoes [3] according to the semi-perimeter, it would be nice to find a bijection between these two classes. A way to do it would be to find a recursive construction for convex polyominoes leading to the same succession rule for square polygons.

\section{Acknowledgements}

The authors are grateful to Simone Rinaldi for interesting discussions and to Gilles Schaeffer for useful comments on a preliminary version of this paper.

\section{References}

[1] Barcucci, E., Del Lungo, A., Pergola, E., Pinzani, R. ECO: a methodology for the Enumeration of Combinatorial Objects. J. Diff. Eq. and App. 5(1999) 435-49.

[2] Boldi, P., Lonati, V., Radicioni, R., Santini, M. The number of convex permutominoes. Rapporto Interno n.311-06, Universita' degli Studi di Milano, available electronically at http://santini.dsi.unimi.it/research/papers.php.

[3] Bousquet-Mélou, M. Habilitation à diriger les recherches, 1996, Université de Bordeaux, available electronically at http://www.labri.fr/perso/bousquet.

[4] Disanto, F., Frosini, A., Pinzani, R., Rinaldi, S. A closed formula for the number of convex permutominoes. The Electronic Journal of Combinatorics, Volume 14(1), 2007, R57.

[5] Flajolet, P., Sedgewick, R. Analytic Combinatorics, Cambridge University Press, to appear, available electronically at http://algo.inria.fr/flajolet.

[6] Mansour, T., Severini, S. Grid polygons from permutations and their enumeration by the kernel method. 19-th Intern. Conf. on Formal Power Series and Algebraic Combinatorics, Center for Combin., Nankai Uni., Tianjin, China, July 2-6, 2007.

[7] Wilf, H. Invited talk at CRM Conference Enumeration and Probabilistic Methods in Combinatorics, June 2007, Barcelona. 